\title{
Nonlinear multicore waveguiding structures with balanced gain and loss
}

\author{
Alejandro J. Martínez, ${ }^{1,2}$ Mario I. Molina, ${ }^{1}$ Sergei K. Turitsyn, ${ }^{3}$ and Yuri S. Kivshar ${ }^{4}$ \\ ${ }^{1}$ Departamento de Física, MSI-Nucleus on Advanced Optics, and Center for Optics and Photonics (CEFOP), \\ Facultad de Ciencias, Universidad de Chile, Santiago, Chile \\ ${ }^{2}$ Oxford Centre for Industrial and Applied Mathematics, Mathematical Institute, University of Oxford, Oxford OX2 6GG, United Kingdom \\ ${ }^{3}$ Aston Institute of Photonic Technologies, Aston University, Birmingham B4 7ET, United Kingdom \\ ${ }^{4}$ Nonlinear Physics Center, Research School of Physics and Engineering, Australian National University, Canberra ACT 0200, Australia
}

(Received 15 May 2014; revised manuscript received 18 November 2014; published 17 February 2015)

\begin{abstract}
We study existence, stability, and dynamics of linear and nonlinear stationary modes propagating in radially symmetric multicore waveguides with balanced gain and loss. We demonstrate that, in general, the system can be reduced to an effective $\mathcal{P} \mathcal{T}$-symmetric dimer with asymmetric coupling. In the linear case, we find that there exist two modes with real propagation constants before an onset of the $\mathcal{P} \mathcal{T}$-symmetry breaking while other modes have always the propagation constants with nonzero imaginary parts. This leads to a stable (unstable) propagation of the modes when gain is localized in the core (ring) of the waveguiding structure. In the case of nonlinear response, we show that an interplay between nonlinearity, gain, and loss induces a high degree of instability, with only small windows in the parameter space where quasistable propagation is observed. We propose a novel stabilization mechanism based on a periodic modulation of both gain and loss along the propagation direction that allows bounded light propagation in the multicore waveguiding structures.
\end{abstract}

DOI: 10.1103/PhysRevA.91.023822

PACS number(s): 42.65.Wi, 42.81.-i, 42.82.Et, 05.45.Yv

\section{INTRODUCTION}

During the last decade, many efforts have been devoted to the study of photonic structures consisting of coupled waveguides with gain and loss $[1,2]$ which offer interesting novel possibilities for shaping optical beams in comparison with traditional conservative or low-loss structures. Many of such structures can be constructed as optical analogs of the quantum potentials possessing the so-called parity-time $(\mathcal{P} \mathcal{T})$ symmetry, where $\mathcal{P}$ stands for the parity operation while $\mathcal{T}$ refers to the time-reversal operation. Quantum systems that are invariant under the combined $\mathcal{P} \mathcal{T}$ operation can have an entirely real eigenvalue spectrum, in spite of being non-Hermitian [3-5]. In this kind of system there is balance between gain and losses, implying a bounded dynamics. In optics this symmetry is realized by considering a complex index of refraction whose real part is an even function of space, whereas its imaginary part is odd.

The first experimental demonstrations of the $\mathcal{P} \mathcal{T}$ symmetric effects in optics were in two-waveguide directional linear couplers [6,7]. Theoretical analysis suggests that such couplers, operating in the nonlinear regime, can be used for the all-optical signal control [8-10]. Arrays of the $\mathcal{P} \mathcal{T}$-symmetric couplers were proposed as a feasible means of control of the spatial beam dynamics, including the formation and switching of spatial solitons [11-13].

Recently, a new theory of coherent propagation and power transfer in low-dimension array of coupled nonlinear waveguides has been suggested by Turitsyn et al. [14,15], where it was demonstrated that in the array with the central core stable steady-state coherent multicore propagation is possible only in the nonlinear regime, with a power-controlled phase matching. This finding opens novel opportunities to explore multicore waveguiding systems, however, it also puts a question about the stability of such waveguiding structures in the presence of gain and loss. We notice that, apart from being an interesting physical system, a multicore optical fiber is now actively studied in the context of the spatial division multiplexing, the technology of transmitting information over separate spatial channels. The spatial division multiplexing enables the up-scaling of the capacity per fiber that is a critical challenge in the modern optical communications $[16,17]$. Multicore optical fibers are also studied in the field of powerful fiber lasers [18], where gain is an important feature of the system. Multicore waveguiding systems may be useful when nonlinear effects limit the power that can be transmitted in a single waveguide. In this case, the multicore waveguiding system can operate in the regime when the light power in each core is below the level of nondesirable physical effects, while coherence is provided by the coupling between the waveguide cores, allowing for the coherent combining of the total power after delivering the signal to destination. Gain and loss are both important in such multicore optical fiber systems.

In this paper, we study both linear and nonlinear dynamics in multicore waveguiding systems suggested earlier in Refs. $[14,15]$, but in the presence of balanced gain and loss, when the system operates as a multicore optical coupler (see Fig. 1). First, we analyze all regimes when the system can be transformed into the $\mathcal{P} \mathcal{T}$-symmetric multicore couplers and study its stability. Then we suggest how to achieve the bounded dynamics in the nonlinear regime by modulating both gain and loss.

The paper is organized as follows. In Secs. II and III we describe a general model and focus on the study of the linear regime finding the critical parameters for gain and loss when the $\mathcal{P} \mathcal{T}$ symmetry breaks. In Sec. IV we discuss the reduction of the multicore coupler to an asymmetric waveguide dimer in the presence of nonlinearity. We find all nonlinear modes and analyze their stability. In Sec. V we study the dynamical evolutions of the modes and discuss their numerical stability, culminating with a proposal to stabilize the system by means of spatially periodic gain and loss. Section VI concludes the paper. 


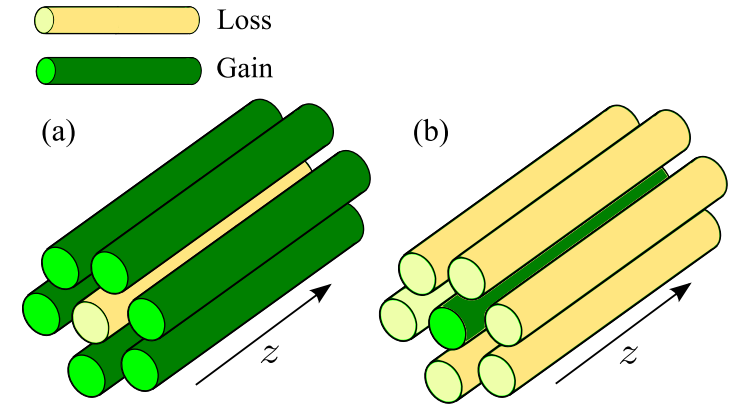

FIG. 1. (Color online) Schematic of the waveguiding structure of a radially symmetric multicore waveguide array with balanced gain and loss.

\section{MODEL}

We consider a multicore waveguide array composed of $N$ identical waveguides arranged in a circular geometry, as shown in Fig. 1. We assume that all waveguides are identical, and they are characterized by the propagation constant $\epsilon_{1}$, with gain/loss parameter $\rho_{1}$. In addition, we include a central waveguide with the propagation constant $\epsilon_{0}$, and gain/loss parameter $\rho_{0}$. The nonlinear parameter $\gamma$ also can be different for the central and peripheral cores, but we assume it to be the same in the presented analysis. In the coupled-modes formalism applied here, we assume the interaction of the nearest neighbors for the waveguides on the ring, and write the evolution equations for the mode amplitudes in the form,

$$
\begin{gathered}
-i \frac{d A}{d z}=\left(\epsilon_{0}+i \rho_{0}\right) A+C_{0} \sum_{j=1}^{N} B_{j}+\gamma|A|^{2} A, \\
-i \frac{d B_{j}}{d z}=\left(\epsilon_{1}+i \rho_{1}\right) B_{j}+C_{1}\left(B_{j+1}+B_{j-1}\right) \\
+C_{0} A+\gamma\left|B_{j}\right|^{2} B_{j},
\end{gathered}
$$

where $A$ is the amplitude of the electric field in the core waveguide, $B_{j}$ is the amplitude in the $j$ th waveguide on the ring, with the conditions $B_{0}=B_{N}$ and $B_{N+1}=B_{1}, \gamma$ being the Kerr nonlinearity coefficient, and $C_{0,1}$ being the coupling coefficients of the modes of different waveguides.

The coupling coefficients $C_{0}$ and $C_{1}$ are not independent. For a circular array of $N$ waveguides, the distance between the nearest-neighbor waveguides in the ring $L$, and the distance from the center core to the ring $R_{0}$, are related by the condition $L=2 R_{0} \sin (\pi / N)$. Now, using the fact that the coupling between different waveguides is due to evanescent optical waves, we can approximate $C_{0} \approx \exp \left(-\mu R_{0}\right)$ and $C_{1} \approx \exp (-\mu L)$, where $\mu$ depends on physical parameters such as geometry of waveguides or their refractive index [19]; thus we obtain

$$
\frac{C_{1}}{C_{0}}=\exp \left\{\mu R_{0}[1-2 \sin (\pi / N)]\right\} .
$$

Note that, for $N<6$, we have $C_{1}<C_{0}$, while for $N \geqslant 6$, we obtain $C_{1} \geqslant C_{0}$, where the equality is satisfied only when $N=6$. We notice that this corresponds to the recently developed seven-core multicore fiber actively studied in optical communication $[16,17]$, where typical examples of the parameters can be found.

\section{LINEAR REGIME}

\section{A. Eigenvalues and linear modes}

First, we consider the linear case when $\gamma=0$. According to Ref. [20], in this case the system described by Eqs. (1) and (2) has only two rotational invariant modes, such that $B_{n}=B$ for all $n$, and these modes are associated with pure real eigenvalues for $|\rho|<\rho_{c}$ :

$$
\lambda^{ \pm}=\epsilon_{1}+2 C-1 \pm \sqrt{N C_{0}^{2}-\rho^{2}}
$$

where $\rho_{0}=\rho=-\rho_{1}$ and $\rho_{c}=\sqrt{N} C_{0}$. The other modes correspond to waves without field in the central guide. As a consequence, their eigenvalues are exactly the eigenvalues of a ring [20], i.e., a one-dimensional chain with periodic boundary condition:

$$
\lambda_{v}=-i \rho+2 C_{1} \cos \left(\frac{2 \pi \nu}{N}\right), \quad v=0,1, \ldots, N-2 .
$$

Moreover, they are organized in a pair of degenerated modes. Figure 2 shows examples of the linear eigenvalues for the case $N=6$. Note that these $N-1$ eigenvalues have an imaginary part equal to $-\rho$, which means that the linear modes associated with them can be written as $B_{n, v}(z)=b_{n} e^{2 i C_{1} \cos \left(\frac{2 \pi v}{N}\right) z} e^{\rho z}$, where $b_{n}$ is the profile of the mode. The dominant behavior is given by the real exponential term $e^{\rho z}$, such that the optical field $B_{n, v}$ either goes to zero for $\rho<0$ or is unbounded for $\rho>0$, as $z \rightarrow \infty$.

From a dynamical point of view, any initial condition of the general system of the form $A(0)=a_{0}$ and $B_{n}(0)=b_{0}$ excites only the modes related to Eq. (4). On the other hand, for a completely arbitrary initial condition, namely, $A(0)=a_{0}$ and $B_{n}(0)=b_{n}$, we have to consider the contribution of each mode in the form,

$$
\begin{aligned}
\left\{A(z), B_{n}(z)\right\}= & \left\{\alpha^{+}, \beta_{n}^{+}\right\} e^{i \lambda^{+} z}+\left\{\alpha^{-}, \beta_{n}^{-}\right\} e^{i \lambda^{-} z} \\
& +\sum_{\nu=0}^{N-2}\left\{\alpha^{\nu}, \beta_{n}^{\nu}\right\} e^{i \lambda_{\nu} z}
\end{aligned}
$$

where the coefficients $\alpha^{+}, \beta^{+}, \ldots$ correspond to the projection of the initial condition over the appropriate eigenvector.

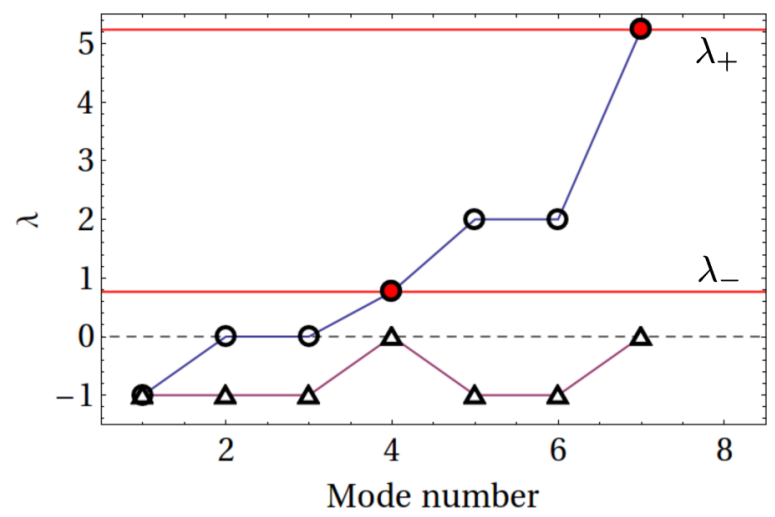

FIG. 2. (Color online) Eigenvalues of Eqs. (1) and (2) for $N=6$, $\gamma=0$, and $\rho=1$. Circles (triangles) show the real (imaginary) part of the eigenvalues. Red lines show the pure real eigenvalues associated with the $\mathcal{P} \mathcal{T}$ symmetry in the reduced system. 
Equations (4) and (5) imply that the dynamic, given by (6), remains bounded only for $-\sqrt{N} C_{0}<\rho<0$, approaching asymptotically a situation where only the modes associated with $\lambda^{ \pm}$have a significant role.

\section{B. Reduction to an effective waveguide dimer}

Since the effective dynamics of the system quickly converges to that of a dimer for $\rho<0$, let us simplify the problem and work with a nondegenerate dimer from the outset $[2,14]$ :

$$
\begin{gathered}
-i \frac{d A}{d z}=\left(\epsilon_{0}+i \rho_{0}\right) A+N C_{0} B, \\
-i \frac{d B}{d z}=\left(\epsilon_{1}+i \rho_{1}\right) B+C_{0} A+2 C_{1} B .
\end{gathered}
$$

Note that solutions described by this reduction are invariant under discrete rotations in $2 \pi n / N(n \in \mathbb{Z})$ respect to the central waveguide.

We pose a solution of the form $A(z)=a \exp (i \lambda z), B(z)=$ $b \exp (i \lambda z)$. This leads to

$$
\begin{gathered}
\left(-\lambda+\epsilon_{0}+i \rho_{0}\right) a+N C_{0} b=0, \\
C_{0} a+\left(-\lambda+\epsilon_{1}+2 C_{1}+i \rho_{1}\right) b=0 .
\end{gathered}
$$

Examination of the determinant of the system reveals that in order to have $\lambda$ real, one needs to impose $\rho_{0}=-\rho_{1}=\rho$ and $\epsilon_{0}-\epsilon_{1}=2 C_{1}$, leading to the propagation constants:

$$
\lambda^{ \pm}=\epsilon_{1}+2 C_{1} \pm \sqrt{N C_{0}^{2}-\rho^{2}} .
$$

Thus, the critical gain and loss parameter value is $\rho_{c}^{2}=N C_{0}^{2}$.

The eigenvectors are given by

$$
\left\{a^{ \pm}, b\right\}=\left\{\frac{i \rho \pm \sqrt{N C_{0}^{2}-\rho^{2}}}{C_{0}}, 1\right\},
$$

and satisfy $\left|a^{ \pm}\right|^{2}=N|b|^{2}$ when $|\rho| \leqslant \rho_{c}$ and, $C_{0}^{2}\left|a^{ \pm}\right|^{2}=(\rho \pm$ $\left.\sqrt{\rho^{2}-N C_{0}^{2}}\right)^{2}|b|^{2}$ when $|\rho|>\rho_{c}$.

At least in the dimer reduction, the requirement over the system parameters leads to a $\mathcal{P} \mathcal{T}$-symmetric dimer, such as in Refs. [9,21]. Thus, even though the Hamiltonian is nonHermitian, its eigenvalues will be in $\mathbb{R}$ until the onset of $\mathcal{P} \mathcal{T}$ symmetry breaking [3]. For any other solution that could not be described by this reduction, the system intrinsically does not satisfy $\mathcal{P} \mathcal{T}$ symmetry. Figure 3 shows the propagation constants as a function of $\rho$, the bifurcation diagram $|a(\rho)|^{2}$, and an example of the intensity distribution of the linear modes.

\section{System dynamics}

We consider the linear dynamics of an arbitrary initial condition: $A(0)=a_{0}, B(0)=b_{0}$. One expands

$$
A(z)=\alpha^{+} \exp \left(i \lambda^{+} z\right)+\alpha^{-} \exp \left(i \lambda^{-} z\right) .
$$

In general, the periodicity of $A(z)$ along $z$ depends on the ratio between $\lambda^{+}$and $\lambda^{-}$. For instance, if $\lambda^{+} / \lambda^{-} \in \mathbb{Z}$ then $A(z)$ will be periodic. Otherwise, it will be aperiodic. Nonetheless,
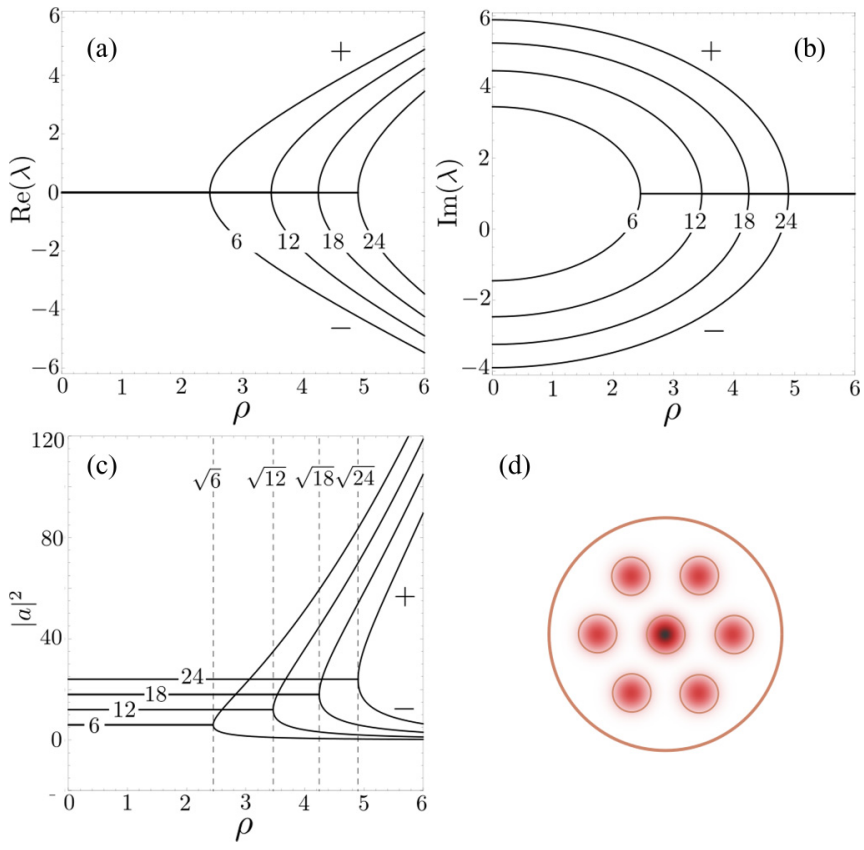

(d)

FIG. 3. (Color online) (a) and (b) The imaginary and real part of the eigenvalues described in Eqs. (9) and (10), respectively. (c) The optical field in the central waveguide, which is given by Eq. (11). (d) An example of the intensity distribution of the linear modes in a system with $N=6$. (a)-(c) Calculated for $N=6,12,18,24$.

the intensity of the field is periodic and given by

$$
|A(z)|^{2}=\left|\alpha^{+}\right|^{2}+\left|\alpha^{-}\right|^{2}+\kappa \sin \left(\left(\lambda^{+}-\lambda^{-}\right) z+\phi\right),
$$

where $\kappa$ and $\phi$ are the amplitude and phase of the periodic oscillation around the average value $\left\langle|A(z)|^{2}\right\rangle_{z}=\left|\alpha^{+}\right|^{2}+\left|\alpha^{-}\right|^{2}$ of the intensity, respectively. Both quantities are functions of the initial condition $\left\{a_{0}, b_{0}\right\}$. Equation (14) means that the intensity is periodic for all $\lambda^{+} \neq \lambda^{-}$. The characteristic propagation constant is given by $\lambda_{c}=\lambda^{+}-\lambda^{-}=2 \sqrt{N C_{0}^{2}-\rho^{2}}$. In particular, when $\lambda^{+}=\lambda^{-}\left(\rho=\rho_{c}\right)$ the intensity remains constant.

One interesting case corresponds to the excitation only at the core, i.e., $a_{0}=1$ and $b_{0}=0$. In this case, the parameters of the expansion are

$$
\begin{aligned}
& \alpha^{+}=\left(\frac{\sqrt{N C_{0}^{2}-\rho^{2}}+i \rho}{2 \sqrt{N C_{0}^{2}-\rho^{2}}}\right), \\
& \alpha^{-}=\left(\frac{\sqrt{N C_{0}^{2}-\rho^{2}}-i \rho}{2 \sqrt{N C_{0}^{2}-\rho^{2}}}\right),
\end{aligned}
$$

and both quantities are singular when $\rho=\rho_{c}$. On the other hand, unlike the case without gain and loss, in general there is no conservation of power $P=|A(z)|^{2}+|B(z)|^{2}$, and the power transfer between the two sites is asymmetrical. This is not a physical problem, rather it is just a mathematical consequence of the reduction. The total power of the entire system, which is conserved in this case, is $P_{N}=|A(z)|^{2}+$ $N|B(z)|^{2}$. 


\section{NONLINEAR REGIME}

\section{A. Structure of nonlinear modes}

Now, we are interested in studying the effects of nonlinearity in the multicore array. As we have seen in the section above, there are only two linear modes which have a real propagation constant within some region of parameters. These correspond to waves that are invariant under discrete rotation, i.e., $B_{n}=$ const. One question that arises from here is the following: Is it possible to continue this mode into the nonlinear regime? To answer this, we extend our nondegenerate dimer analysis to the case where nonlinearity is considered. This is based on the fact that we do not expect nonlinearity to induce a breaking of the spatial symmetry of the linear modes, at least when nonlinear effects are enough small, thus we look for solutions that are still having $B_{n}=$ const. In the presence of nonlinear effects, the equations read

$$
\begin{gathered}
-i \frac{d a}{d z}=\left(\epsilon_{0}+i \rho_{0}\right) a+N C_{0} b+\gamma|a|^{2} a, \\
-i \frac{d b}{d z}=\left(\epsilon_{1}+i \rho_{1}\right) b+C_{0} a+2 C_{1} b+\gamma|b|^{2} b .
\end{gathered}
$$

Systems described by equations like (18) have been extensively studied in the past, mainly in the case where the coupling between $a$ and $b$ is nondirectional (see, e.g., [22]); i.e., $N=1$, however, in our case we consider a directional coupling between the nonlinear couplers, which naturally appears due to the dimer reduction of the multicore structure. A stationary state solution $a(z)=a \exp (i \lambda z), b(z)=b \exp (i \lambda z)$, leads to the equations,

$$
\begin{gathered}
\left(-\lambda+\epsilon_{0}+i \rho+\gamma|a|^{2}\right) a+N C_{0} b=0, \\
C_{0} a+\left(-\lambda+\epsilon_{1}+2 C_{1}-i \rho+\gamma|b|^{2}\right) b=0 .
\end{gathered}
$$

The transformation, $\left\{m^{+}, \lambda^{+}, \rho, \gamma\right\} \rightarrow\left\{m^{-}, \lambda^{-},-\rho,-\gamma\right\}$, leaves Eqs. (19) and (20) invariant. Thus, we will analyze only the case with self-focusing nonlinearity $(\gamma>0)$. These above equations have exactly nine complex solutions, one of them being the zero (trivial) solution. The other solutions are organized in pairs with an identical relation between $P_{N}$ and $\lambda$. Figure 4 shows the power vs propagation constant diagram for these modes, as well as their real and imaginary parts as functions of the propagation constant $\lambda$. We note that there are four nonlinear branches that emerge exactly from the propagation constant associated with the linear modes described by Eq. (12).

In order to simplify the description of these solutions, we introduce a shift in the propagation constant $\lambda \rightarrow \lambda+$ $\epsilon_{0}=\lambda+\left(\lambda^{+}+\lambda^{-}\right) / 2$, with $\epsilon_{0}=\epsilon_{1}+2 C_{1}$. Thus, Eqs. (19) and (20) read

$$
\lambda \vec{v}=\left(\begin{array}{cc}
i \rho+\gamma|a|^{2} & N C_{0} \\
C_{0} & -i \rho+\gamma|b|^{2}
\end{array}\right) \vec{v},
$$

where $\vec{v}=(a, b)^{T}$, and $T$ denotes the transpose.

Equation (21) has a phase invariance, i.e., it is invariant under a global phase shift $\vec{v} \rightarrow \vec{v} e^{i \theta}$. This is connected with the fact that the conservation of the power $P_{N}$ is a necessary condition in order to have stationary fields. Actually, it is easy to show that to avoid fluctuations in the power along
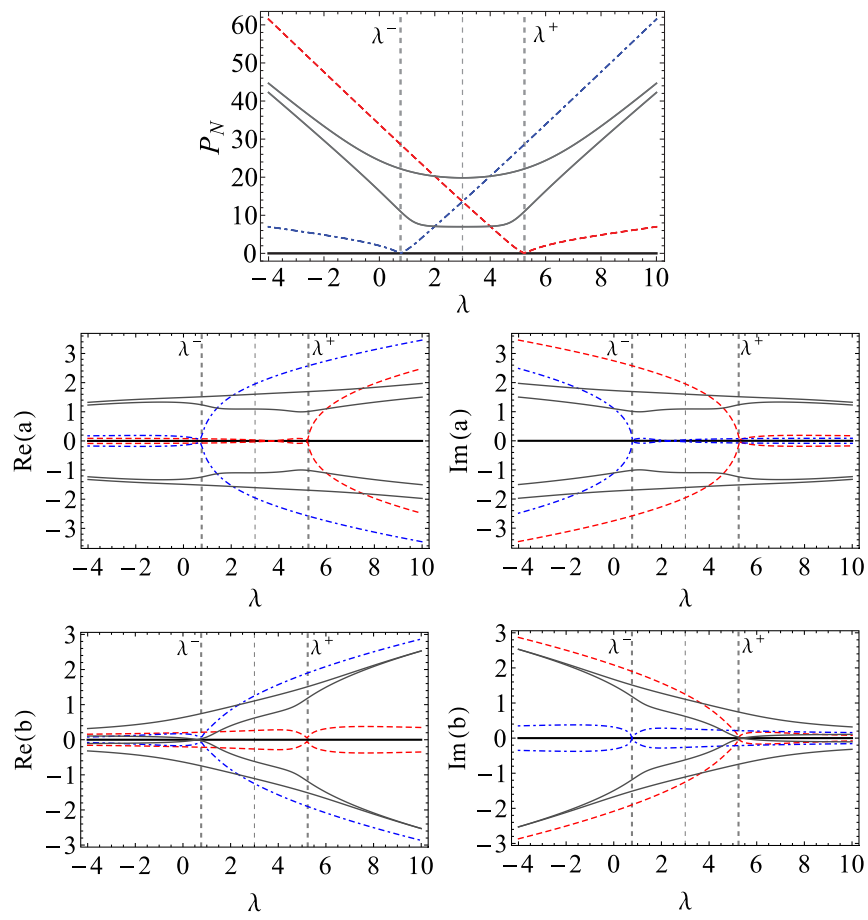

FIG. 4. (Color online) $P_{N}, \operatorname{Re}\{a\}, \operatorname{Re}\{b\}, \operatorname{Im}\{a\}$, and $\operatorname{Im}\{b\}$ associated with the nine solutions of Eqs. (19) and (20) as function of the propagation constant $\lambda$. Red and blue lines denote the modes described by Eq. (23). Thick vertical lines denote the propagation constants $\lambda^{ \pm}$, while thin vertical lines are related to $\left(\lambda^{+}+\lambda^{-}\right) / 2$.

the propagation direction, $P_{N}$ must satisfy

$$
\frac{d P_{N}}{d z}=-2 \rho\left(|a|^{2}-N|b|^{2}\right)=0,
$$

which means that solutions of (19) and (20) must satisfy $|a|^{2}=N|b|^{2}$. This relation can equivalently be derived directly from Eqs. (19) and (20) through imposing condition of real eigenvalues $\operatorname{Im}(\lambda)=0$. Thus, there are pairs of solutions of the form $\pm\{a, b\}$ as shown in Fig. 4 .

Moreover, Eqs. (19) and (20) represent a nonlinear spectral problem with solutions $a, b$, and $\lambda$ being functions of $N, \epsilon_{0,1}, C_{0,1}, \gamma$, and $\rho$. Let us denote $\operatorname{Re}(\lambda)=\lambda_{R}$, $\operatorname{Im}(\lambda)=\lambda_{I}$, and introduce $\Gamma=b / a$. Now, we look for stationary solutions assuming that $|a|^{2}=N|b|^{2}$ is satisfied and $P_{N}=|a|^{2}+N|b|^{2}=$ const, thus we get from Eqs. (19) and (20) that $\Gamma_{I}=\operatorname{Im}(\Gamma)=-\rho /\left(N C_{0}\right)$ and $\Gamma_{R}=\operatorname{Re}(\Gamma)=$ $\pm \sqrt{1 / N-\rho^{2} /\left(N^{2} C_{0}^{2}\right)}$. Thereby, the nonlinear solutions read

$$
m^{ \pm}=\left\{a^{ \pm}, b\right\}=\sqrt{\frac{P_{N}}{2 N}}\left\{\frac{i \rho \pm \sqrt{N C_{0}^{2}-\rho^{2}}}{C_{0}}, 1\right\},
$$

and the propagation constants are

$$
\lambda_{\gamma}^{ \pm}=\epsilon_{0}+\gamma \frac{P_{N}}{2} \pm \sqrt{N C_{0}^{2}-\rho^{2}},
$$

which correspond to the nonlinear continuation of the linear modes given by Eq. (12). These naturally satisfy $\left|a^{ \pm}\right|^{2}=$ $N|b|^{2}$ (when $|\rho|<\rho_{c}$ ) by construction. Furthermore, the $\mathcal{P} \mathcal{T}$ symmetry-breaking critical gain and loss parameter is the 
same as before, $\rho_{c}^{2}=N C_{0}^{2}$. Additionally, we note that while nonlinearity induces a shift in the propagation constant of these modes, their envelope remains unchanged, except by a constant factor that depends on the total power and the number of waveguides in the multicore array.

\section{B. Stability analysis}

To examine the linear stability of the nonlinear modes given by Eq. (23), we introduce small perturbations and write the amplitudes in the form, $A(z) \rightarrow\left(a+\delta_{0}(z)\right) \exp (i \lambda z)$ and $B \rightarrow\left(b+\delta_{1}(z)\right) \exp (i \lambda z)$. After inserting this into Eqs. (17) and (18), we obtain in the first order in $\delta_{0}, \delta_{1}$ the following linear equations,

$$
\begin{aligned}
-i \frac{d \delta_{0}}{d z}= & \left(-\lambda+\epsilon_{0}+i \rho\right) \delta_{0}+N C_{0} \delta_{1} \\
& +\gamma a^{2} \delta_{0}^{*}+2 \gamma|a|^{2} \delta_{0}, \\
-i \frac{d \delta_{1}}{d z}= & \left(-\lambda+\epsilon_{1}+2 C_{1}-i \rho\right) \delta_{1}+C_{0} \delta_{0} \\
& +\gamma b^{2} \delta_{1}^{*}+2 \gamma|b|^{2} \delta_{1} .
\end{aligned}
$$

Next, we split $\delta_{0}$ and $\delta_{1}$ into their real and imaginary parts: $\delta_{0}=\alpha_{0}+i \beta_{0}, \delta_{1}=\alpha_{1}+i \beta_{1}$. We also decompose $a=x_{0}+$ $i y_{0}, b=x_{1}+i y_{1}$. After replacing into Eqs. (25) and (26) and after defining $\vec{w}=\left(\alpha_{0}, \beta_{0}, \alpha_{1}, \beta_{1}\right)^{T}$, we obtain an equation of the form,

$$
\frac{d}{d z} \vec{w}=\mathbf{M} \vec{w}
$$

where $\mathbf{M}=\left\{M_{i, j}\right\}$ is a $4 \times 4$ matrix with the components $M_{1,3}=M_{2,4}=M_{3,1}=M_{4,2}=0$, and

$M_{1,1}, M_{2,2}=-\rho \mp 2 \gamma x_{0} y_{0}$

$M_{1,2}, M_{2,1}= \pm \lambda \mp \epsilon_{0}+\gamma\left(x_{0}^{2}-y_{0}^{2}\right) \mp 2 \gamma\left(x_{0}^{2}+y_{0}^{2}\right)$,

$M_{1,4}, M_{2,3}=\mp N C_{0}$,

$M_{3,2}, M_{4,1}=\mp C_{0}$,

$M_{3,3}, M_{4,4}=\rho \mp 2 \gamma x_{1} y_{1}$,

$M_{3,4}, M_{4,3}= \pm \lambda \mp \epsilon_{1}-2 C_{1}+\gamma\left(x_{1}^{2}-y_{1}^{2}\right) \mp 2 \gamma\left(x_{1}^{2}+y_{1}^{2}\right)$.

The stability condition requires that the real part of all eigenvalues $\{v\}$ of $\mathbf{M}$ be negative. Thus, we define the instability gain $g$ as the real part of the eigenvalue with the largest positive real part.

For the simple case with no gain and loss, it is possible to obtain the eigenvalues in closed form:

$$
\begin{gathered}
v_{1}^{+}=-4 N C_{0}^{2}+2 \gamma(N+1) \sqrt{N} C_{0}, \\
v_{2}^{+}=0,
\end{gathered}
$$

for the $m^{+}$mode $\{\sqrt{N}, 1\}$, and

$$
\begin{gathered}
v_{1}^{-}=-4 N C_{0}^{2}-2 \gamma(N+1) \sqrt{N} C_{0}, \\
v_{2}^{-}=0,
\end{gathered}
$$

for the $m^{-}$mode $\{-\sqrt{N}, 1\}$. We conclude that the $m^{-}$ mode is stable while the $m^{+}$mode is stable provided $\gamma<$ $2 \sqrt{N} C_{0} /(N+1)$. This defines a critical nonlinearity, which
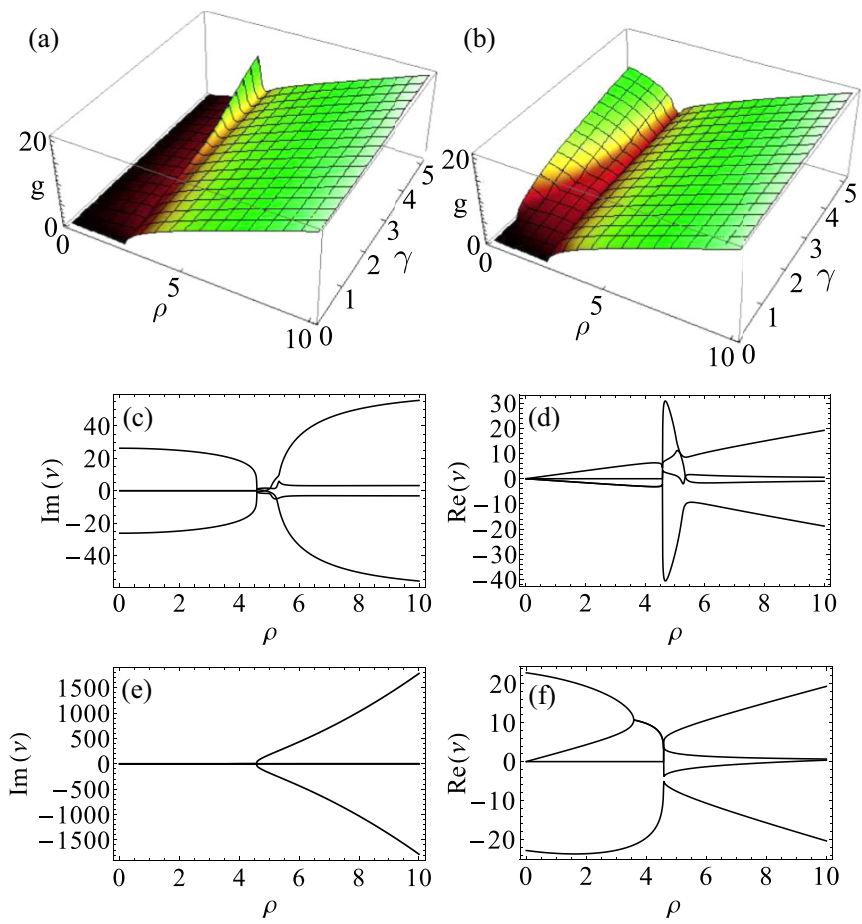

FIG. 5. (Color online) (a) and (b) The instability gain $g$ as a function of $\rho>0$ and $\gamma$ for $N=6$ for the $m^{-}$and $m^{+}$mode, respectively. (c)-(f) The eigenvalues $\{v\}$ as functions of $\rho(0 \leqslant \rho \leqslant 10)$ for $\gamma=3$, and $N=21$. (c) and (d) The imaginary and real part of $\{v\}$ for the $m^{-}$mode, respectively, and (e) and (f) for the $m^{+}$mode.

depends on the size of the system, given by

$$
\gamma_{c}=\frac{2 \sqrt{N} C_{0}}{(N+1)}
$$

However, numerical examination of the behavior of the instability gain suggests that as soon as $\rho \neq 0$ the nonlinear system becomes unstable. For $\rho>0$, Figs. 5(a) and 5(b) show the behavior of $g$ as a function of $\rho$ and $\gamma$. For both modes, the interplay between nonlinearity and gain and loss causes destabilization of the modes. In the case of the $\mathrm{m}^{+}$mode, this destabilization is bounded, at least for $\rho<\rho_{c}$ and $\gamma<\gamma_{c}$. Out of this region, there are bubblelike domains where $g$ increases abruptly. Nevertheless, in the case of the $m^{-}$mode, there are two main regions: $\rho \lesssim \rho_{c}$, and $\rho \gtrsim \rho_{c}$. In the former the mode is weakly unstable; otherwise, in the latter the mode is highly unstable. Both regions are separated by a peak of instability. Figures 5(c)-5(f) show the full outlook associated with the distribution of eigenvalues of $M$ as a function of $\rho$. Moreover, Fig. 6 shows the same as Fig. 5, but considering $\rho<0$.

The main result of this section is that, for a nonlinear dimer in a stable regime, the addition of any amount of gain and loss will destabilize the system. This feature will still hold for the general case, as shown in the next section.

\section{Stability analysis in a general system}

Let us consider the stability problem for the general stationary modes $\left\{A^{s},\left\{B_{n}^{s}\right\}_{n=1}^{N}\right\}$ derived from Eqs. (1) and (2). We introduce a linear perturbation of the form $A(z)=\left(a^{s}+\right.$ $\left.\delta_{0}(z)\right) e^{i \lambda z}$, and $B_{n}(z)=\left(b_{n}^{s}+\delta_{n}(z)\right) e^{i \lambda z}$, with $a^{s}=x_{0}+i y_{0}$, 

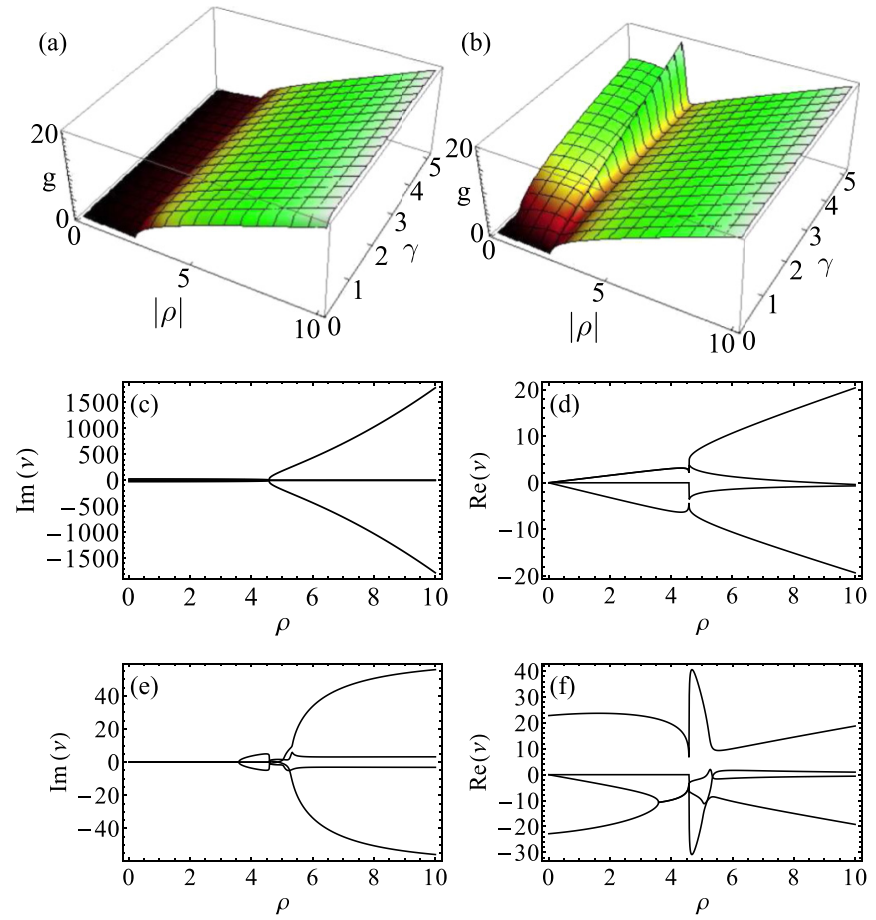

FIG. 6. (Color online) (a) and (b) The instability gain $g$ as a function of $\rho<0$ and $\gamma$ for $N=6$ for the $m^{-}$and $m^{+}$modes, respectively. (c)-(f) The eigenvalues $\{v\}$ vs $|\rho|(-10 \leqslant \rho \leqslant 0)$ for $\gamma=3$ and $N=21$. (c) and (d) The imaginary and real parts of $\{v\}$ for the mode $\mathrm{m}^{-}$, respectively, and (e) and (f) for the mode $\mathrm{m}^{+}$.

$b_{n}^{s}=x_{n}+i y_{n}$, and $\delta_{n}(z)=\alpha_{n}(z)+i \beta_{n}(z)$. Then, we obtain the following linear system for the perturbation:

$$
\begin{gathered}
\frac{d \alpha_{0}}{d z}=-\left(\rho_{0}+2 \gamma x_{0} y_{0}\right) \alpha_{0}-C_{0} \sum_{j=1}^{N} \beta_{j} \\
+\left[\lambda-\epsilon_{0}-\gamma\left(x_{0}^{2}+3 y_{0}^{2}\right)\right] \beta_{0}, \\
\frac{d \beta_{0}}{d z}=\left(2 \gamma x_{0} y_{0}-\rho_{0}\right) \beta_{0}+C_{0} \sum_{j=1}^{N} \alpha_{j} \\
\quad+\left[\epsilon_{0}-\lambda+\gamma\left(3 x_{0}^{2}+y_{0}^{2}\right)\right] \alpha_{0}, \\
\frac{d \alpha_{n}}{d z}=-\left(\rho_{1}+2 \gamma x_{n} y_{n}\right) \alpha_{n}-C_{1}\left(\beta_{n+1}+\beta_{n-1}\right) \\
-C_{0} \beta_{0}+\left[\lambda-\epsilon_{1}-\gamma\left(x_{n}^{2}+3 y_{n}^{2}\right)\right] \beta_{n}, \\
\frac{d \beta_{n}}{d z}=\left(2 \gamma x_{n} y_{n}-\rho_{1}\right) \beta_{n}+C_{1}\left(\alpha_{n+1}+\alpha_{n-1}\right) \\
\times C_{0} \alpha_{0}+\left[\epsilon_{1}-\lambda+\gamma\left(3 x_{n}^{2}+y_{n}^{2}\right)\right] \alpha_{n} .
\end{gathered}
$$

Figure 7 shows the numerical results for the instability gain obtained from this analysis. We note that the behavior of $g$ as a function of $\rho$ and $\gamma$ is qualitatively the same as the one obtained using the analysis for the reduced system. In fact, the instability bubbles are still separated by a neighborhood around $\rho=\rho_{c}$. Furthermore, for the $m^{-}$mode, we obtain that there is a small global increment of the parameter $g$, which suggests higher levels of instability with respect to the reduced description.
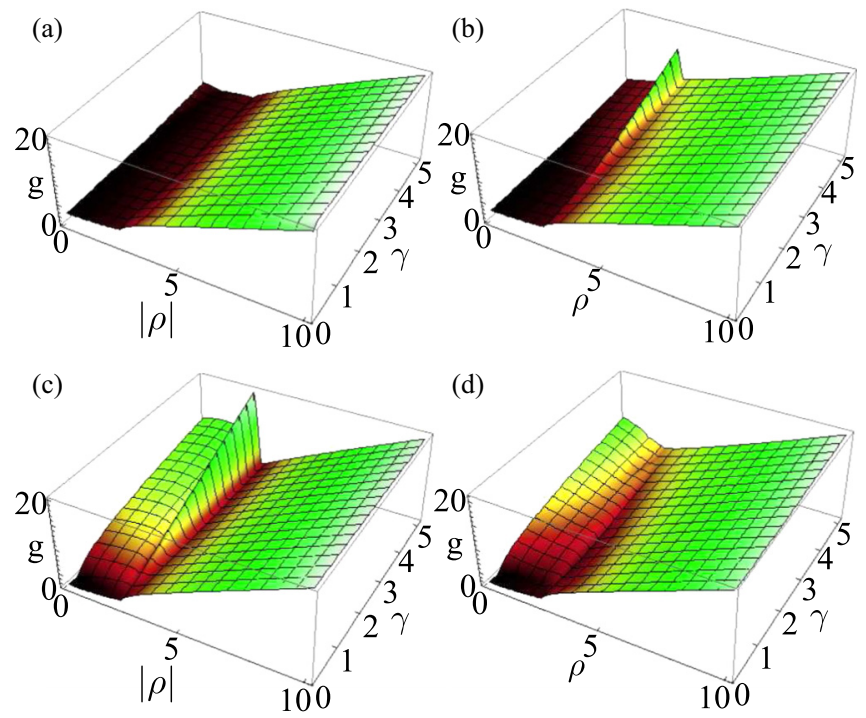

FIG. 7. (Color online) Instability gain $g$ as a function of $\rho$ and $\gamma$ for $N=6$ using Eqs. (31)-(34). (Left) $\rho<0$ and (right) $\rho>0$. (a) and (b) For the mode $m^{-}$; (c) and d) for the mode $m^{+}$.

Nonetheless, numerically we found that $m^{-}$behaves "stable" in a bigger set of the parameter space than $m^{+}$does, since the stability region for $m^{+}$is bounded by $\rho_{c}$ and $\gamma_{c}$. This will be discussed in the next section.

We can therefore conclude that a small addition of gain and loss will destabilize the nonlinear multicore array.

\section{NONLINEAR DYNAMICS AND MODE STABILIZATION}

We now study numerically the system dynamics described by Eqs. (1) and (2) in some interesting cases. First, we consider the case $\rho=0$. Figure 8 shows the evolution for different initial conditions. Among these, we show the propagation of the nonlinear modes $m^{ \pm}$. The $m^{-}$mode displays a stable propagation along $z$, while the $m^{+}$mode shows a modulation along $z$ as a consequence of an energy exchange between the core and the ring. On the other hand, when the initial excitation
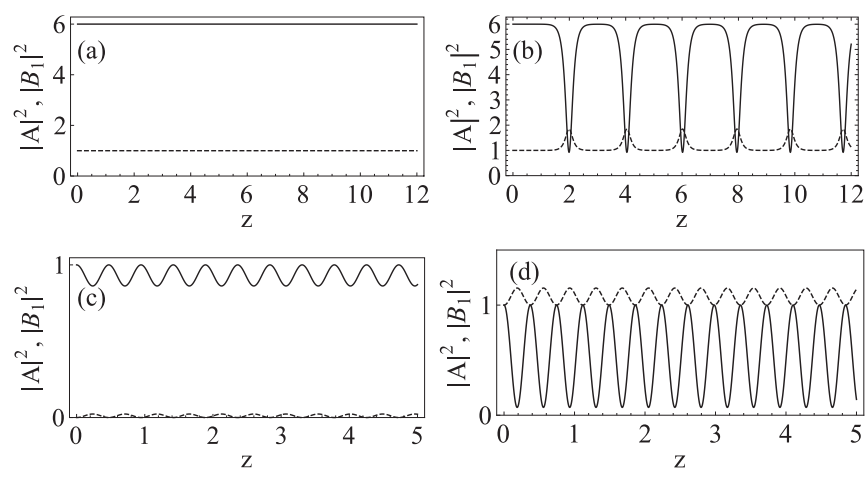

FIG. 8. Examples of numerical integration of Eqs. (1) and (2) in the nonlinear regime $(\gamma=3)$ for $N=6$ and $\rho=0$. Continuous and dashed lines show $|A(z)|^{2}$ and $\left|B_{1}(z)\right|^{2}$, respectively. Shown are (a) $\mathrm{m}^{-}$mode, (b) $\mathrm{m}^{+}$mode, (c) $A(0)=1$ and $B_{j}(0)=0$, and (d) $A(0)=B_{j}(0)=1$. 

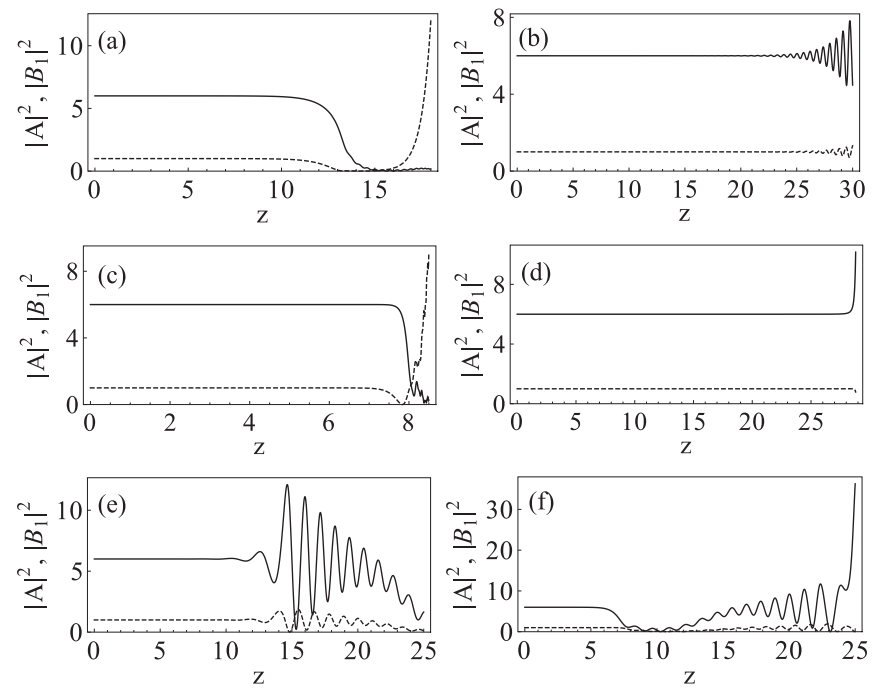

FIG. 9. Examples of numerical integration of Eqs. (1) and (2) in the nonlinear regime for $N=6$ and $\rho \neq 0$. Continuous and dashed lines show $|A(z)|^{2}$ and $\left|B_{1}(z)\right|^{2}$, respectively. Left and right columns correspond to the cases $\rho>0$ and $\rho<0$, respectively. Shown is the mode $m^{-}$in (a)-(d), and the mode $m^{+}$in (e) and (f). Parameters are in (a) and (b) $|\rho|=1$ and $\gamma=3$, (c) and (d) $|\rho|=\rho_{c}=\sqrt{6}$ and $\gamma=3$, and (e) and (f) $|\rho|=1$ and $\gamma=0.5<\gamma_{c}$.

is either only at the core or in the whole array, the field oscillates periodically between the core and ring.

However, as soon as either gain or loss do not vanish, the evolution of the amplitudes $|A(z)|^{2}$ and $\left|B_{n}(z)\right|^{2}$ becomes unstable. In this case, the dynamics of almost any initial conditions shows an early divergence (for small $z$ ), except for the mode $\mathrm{m}^{-}$, whose dynamics resembles a self-trapping state, up to the onset of instability. Figure 9 shows some examples of the propagation for the $\mathrm{m}^{-}$mode for different parameters. Thus, simultaneous presence of nonlinearity, gain, and loss leads to destabilization of the system dynamics. Figure 9 shows that the optical field diverges sooner for $\rho>0$ than for $\rho<0$. This is a generic behavior of the system for almost any initial condition, as shown below.

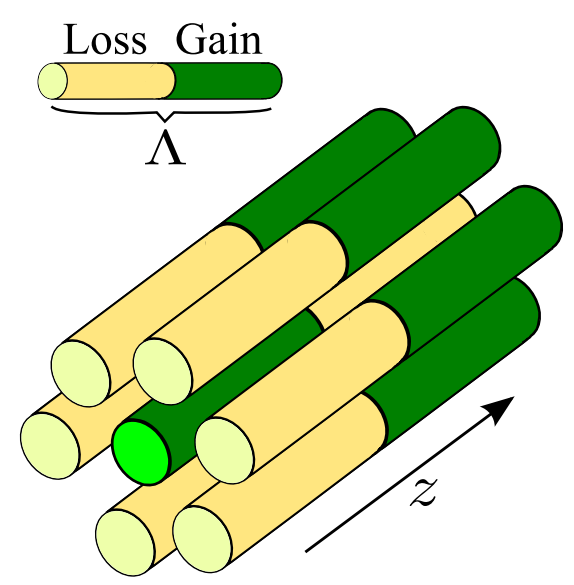

FIG. 10. (Color online) Multicore configuration with a squarelike periodic modulation of gain and loss along the propagation direction $z$.

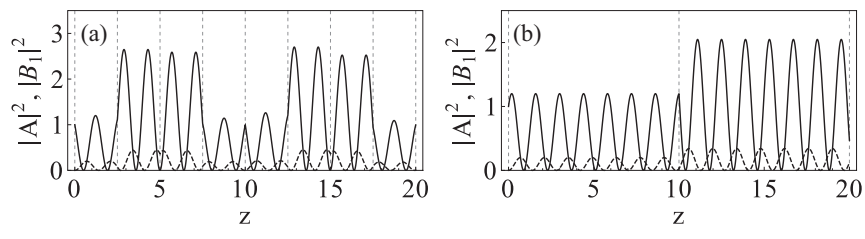

FIG. 11. Examples of numerical integration of Eqs. (1) and (2) in the linear regimen $(\gamma=0)$ for $N=6$ and $|\rho|=1$, with a squarelike modulation of $\rho$. Continuous and dashed lines mark $|A(z)|^{2}$ and $\left|B_{1}(z)\right|^{2}$, respectively. Left and right columns correspond to the cases $\rho>0$ and $\rho<0$, respectively. The initial conditions are $A(0)=1$ and $B_{j}(0)=0$ in both the cases. (a) $\Lambda=5$; (b) $\Lambda=20$.

As we have seen, the nonlinear multicore system becomes unstable in the presence of gain and loss. What this means is that energy does not dissipate and diffuse away at the same rate that it accumulates. To alleviate this problem we will introduce gain and loss terms whose sign will change periodically [23,24]. As a result, the spatial average of gain and loss terms will vanish, and the stable dynamics may be recovered. More specifically, we now take the gain and loss parameter to be a function of $z$ (see Fig. 10). This kind of modulation has been implemented before by different authors, such as [25,26], in the context of waveguide arrays, leading to an increased transport regime, and an unidirectional fractional phase exchange. We use here a squarelike periodic modulation given by $\rho \rightarrow \rho(z)=\eta$ square $(\Lambda z)$ [26], where $\Lambda$ represents the period, and $\eta$ the intensity of the gain and loss. The divergences of optical fields depend on both gain and loss parameter $\rho$, and have the general form $\sim e^{\operatorname{sgn}\{\rho\} v z}$, where sgn is the signum function, and $v$ is a measure of the strength of the divergence. Thus, when the sign of $\rho$ along $z$ changes, the dynamics goes from an exponential increment to an exponential decrement, or vice versa.

Examples of the effects of this modulation on the dynamics are shown in Figs. 11 and 12 for both linear and nonlinear regimes, respectively. In the linear regime, the propagation constants are not modified, and we only observe effects in the
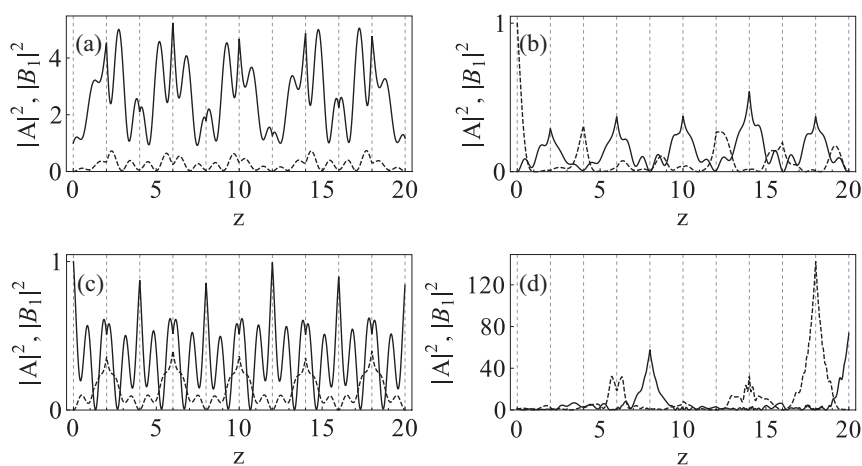

FIG. 12. Examples of numerical integration of Eqs. (1) and (2) in the nonlinear regime $(\gamma=1)$ for $N=6$ for $|\rho|=1$, considering a squarelike modulation of $\rho$ with $\Lambda=4$. Continuous and dashed lines are associated with $|A(z)|^{2}$ and $\left|B_{1}(z)\right|^{2}$, respectively. The parameters are as follows: (a) $\rho<0, A(0)=1, B_{j}(0)=0$; (b) $\rho<0, A(0)=0$, $B_{1}(0)=1, B_{j>1}(0)=0$; (c) $\rho>0, A(0)=1, B_{j}(0)=0$; and (d) $\rho>0, A(0)=1, B_{1}(0)=1, B_{j>1}(0)=0$. 
amplitude of the waves. How amplitude will respond depends on the ratio between the propagation constant $\lambda$ and period $\Lambda$ of the modulation. On the other hand, in the nonlinear regime we found that the modulation induces an effective dynamic stabilization, where the field remains bounded along the propagation direction. It is interesting to notice that this effect is also present for initial conditions that do not have any particular symmetry, such as the case of Figs. 12(b) and 12(d).

\section{CONCLUSIONS}

We have studied the dynamics of nonlinear multicore waveguiding structures with balanced gain and loss. For the linear regime, we have shown that the bounded dynamics can be observed in the limit of an effective waveguide dimer and when gain is placed in the core of the multicore structure. Thus, the dynamics can be reduced to that of an effective $\mathcal{P} \mathcal{T}$ symmetric waveguide dimer with an asymmetric coupling. Within this reduction, we have computed the eigenvalues and eigenvectors of the structure and found the critical value of gain and loss for an onset of the $\mathcal{P} \mathcal{T}$-symmetry-breaking instability.

In the nonlinear case, we have found and analyzed nine stationary modes, four of which bifurcate from the linear modes. For these modes, we have found that the propagation constant remains the same as for the linear case but it gets shifted, while the eigenvectors are found to have the same envelope as in the linear case, except by a constant factor which depends on the power and the number of waveguides in the system. For these modes, we have conducted the stability analysis, and found that the modes are all unstable in the presence of nonlinearity, gain, and loss. We have found a critical parameter for balanced gain and loss separating the regions of low and high instability. Furthermore, we have revealed that the stabilization of nonlinear modes can be achieved by applying a spatially periodic modulation of gain and loss, and we have examined the corresponding bounded dynamics for all initial conditions. We believe that an experimental realization of these findings might help resolve issues related to optical energy transport through multicore waveguiding structures.

\section{ACKNOWLEDGMENTS}

This work was supported in part by Fondo Nacional de Ciencia y Tecnología (Grant 1120123), Programa Iniciativa Científica Milenio (Grant P10-030-F), Programa de Financiamiento Basal (Grant FB0824) and the Australian Research Council. In addition, A.J.M. acknowledges support from Comisión Nacional de Investigación Científica y Tecnológica (Fellowship BCH72130485/2013).
[1] A. Ruschhaupt, F. Delgado, and J. G. Muga, J. Phys. A 38, L171 (2005).

[2] R. El-Ganainy, K. G. Makris, D. N. Christodoulides, and Z. H. Musslimani, Opt. Lett. 32, 2632 (2007).

[3] C. M. Bender and S. Boettcher, Phys. Rev. Lett. 80, 5243 (1998).

[4] C. M. Bender, D. C. Brody, and H. F. Jones, Am. J. Phys. 71, 1095 (2003).

[5] C. M. Bender, Rep. Prog. Phys. 70, 947 (2007).

[6] A. Guo, G. J. Salamo, D. Duchesne, R. Morandotti, M. VolatierRavat, V. Aimez, G. A. Siviloglou, and D. N. Christodoulides, Phys. Rev. Lett. 103, 093902 (2009).

[7] C. E. Rüter, K. G. Makris, R. El-Ganainy, D. N. Christodoulides, M. Segev, and D. Kip, Nat. Phys. 6, 192 (2010).

[8] H. Ramezani, T. Kottos, R. El-Ganainy, and D. N. Christodoulides, Phys. Rev. A 82, 043803 (2010).

[9] A. A. Sukhorukov, Z. Y. Xu, and Yu. S. Kivshar, Phys. Rev. A 82, 043818 (2010).

[10] N. V. Alexeeva, I. V. Barashenkov, K. Rayanov, and S. Flach, Phys. Rev. A 89, 013848 (2014).

[11] S. V. Dmitriev, A. A. Sukhorukov, and Yu. S. Kivshar, Opt. Lett. 35, 2976 (2010).

[12] M. C. Zheng, D. N. Christodoulides, R. Fleischmann, and T. Kottos, Phys. Rev. A 82, 010103 (2010).

[13] S. V. Suchkov, B. A. Malomed, S. V. Dmitriev, and Yu. S. Kivshar, Phys. Rev. E 84, 046609 (2011).
[14] S. K. Turitsyn, A. M. Rubenchik, M. P. Fedoruk, and E. Tkachenko, Phys. Rev. A 86, 031804 (2012).

[15] A. M. Rubenchik, E. V. Tkachenko, M. P. Fedoruk, and S. K. Turitsyn, Opt. Lett. 38, 4232 (2013).

[16] B. Zhu, T. F. Taunay, M. F. Yan, J. M. Fini, M. Fishteyn, E. M. Monberg, and F. V. Dimarcello, Opt. Express 18, 11117 (2010).

[17] F. Y. M. Chan, A. P. T. Lau, and H.-Ya. Tam, Opt. Express 20, 4548 (2012).

[18] B. M. Shalaby, V. Kermene, D. Pagnoux, A. DesfargesBerthelemot, A. Barthelemy, A. Popp, M. Abdou Ahmed, A. Voss, and T. Graf, App. Phys. B: Lasers and Optics 100, 859 (2010).

[19] Allan W. Snyder, J. Opt. Soc. Amer. 62, 1267 (1972).

[20] K. Hizanidis, S. Droulias, I. Tsopelas, N. K. Efremidis, and D. N. Christodoulides, Int. J. Bifurcation Chaos 16, 1739 (2006).

[21] N. V. Alexeeva, I. V. Barashenkov, A. A. Sukhorukov, and Yu. S. Kivshar, Phys. Rev. A 85, 063837 (2012).

[22] Y. Chen, A. W. Snyder, and D. N. Payne, IEEE J. Quant. Electro. 28, 239 (1992).

[23] F. Kh. Abdullaev, V. V. Konotop, M. Ögren, and M. P. Sørensen, Opt. Lett. 36, 4566 (2011).

[24] X. Luo, J. Huang, H. Zhong, X. Qin, Q. Xie, Yu. S. Kivshar, and C. Lee, Phys. Rev. Lett. 110, 243902 (2013).

[25] R. El-Ganainy, K. G. Makris, and D. N. Christodoulides, Phys. Rev. A 86, 033813 (2012).

[26] G. Della Valle and S. Longhi, Phys. Rev. A 87, 022119 (2013). 